This work is licensed under a Creative Commons Attribution 4.0 International License.

Ovaj rad dostupan je za upotrebu pod licencom Creative Commons Imenovanje 4.0 međunarodna.

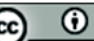

Michael FASSEL

Philosophische Fakultät, Universität Siegen, Deutschland

Adolf-Reichwein-Str. 2, 57068 Siegen

michael.fassel@uni-siegen.de
UDK 821.112.2.09 Jens, T. DOI:https://doi.org/10.29162/ANAFORA.v8i2.10

Wissenschaftliche Mitteilung Preliminary communication

Erhalten am 27. 5. 2021

Received: 27 May 2021

Angenommen am 23. 9. 2021

Accepted: 23 September 2021

\title{
TABUBRUCH ALS STRATEGIE? ZUR DEMENZ- DARSTELLUNG IN TILMAN JENS' DEMENZ. ABSCHIED VON MEINEM VATER (2009)
}

\section{Zusammenfassung}

Tilman Jens schildert in Demenz. Abschied von meinem Vater (2009) den geistigen Verfall seines Vaters Walter Jens. Im Rahmen dieser autobiographischen Erzählung stilisiert sich der Autor als Tabu-Brecher, da der Demenz, so Jens, ein stigmatisierender Moment innewohne. Fraglich ist, ob Tilman Jens tatsächlich als Vorreiter der deutschsprachigen Demenzliteratur betrachtet werden kann. Die Reaktionen des überregionalen Feuilletons mögen diese zentrale These auf den ersten Blick bestätigen. Der vorliegende Beitrag setzt sich mit Jens' Text sowie den Verrissen des Feuilletons genauer auseinander und möchte zeigen, dass nicht die Thematisierung der Demenz, sondern vielmehr die Darstellungsweise der prominenten Persönlichkeit Walter Jens ethische Bedenken auslöst. Vor diesem Hintergrund wird einleitend das Tabu-Verständnis nach Sigmund Freud für die Analyse fruchtbar gemacht, um erstens festzustellen, ob und inwiefern Jens ein Tabu bricht. Darauf aufbauend geht der Beitrag zweitens der These nach, dass vor allem die Schilderung des krankheitsbedingten Gedächtnisverlustes eines prominenten Intellektuellen wie Walter Jens der eigentliche Skandal ist, während vergleichbare Demenznarrative lobenswert be- 
sprochen werden. Im Licht des dementen Walter Jens erweist sich Thomas Manns Überlegung, es komme darauf an, wer krank sei, als ausgesprochen aktuell.

Schlüsselwörter: Demenz, Tabu, Krankheit, Pathographie, Vater, Tilman Jens. Demenz. Abschied von meinem Vater

\section{Einleitung}

Wenn Tilman Jens die Krankheit Demenz in Demenz. Abschied von meinem Vater (2009) als Tabu bezeichnet und gleichzeitig über seinen dementen Vater schreibt, stilisiert sich der Autor als Tabubrecher. Man könnte annehmen, die empörten Stimmen aus dem überregionalen Feuilleton gäben ihm recht. Jens wird unter anderem unterstellt, dass er literarischen Vatermord begehe bzw. seinen noch lebenden Vater lebendig begrabe (Ueding). Denn hinter dieser Vaterfigur verbirgt sich keine geringere Persönlichkeit als Walter Jens, der bekannte Rhetorik-Professor aus Tübingen. Der vorliegende Beitrag setzt sich kritisch mit dem Tabubegriff auseinander und verfolgt die These, dass Tilman Jens keineswegs ein Tabu gebrochen hat, indem er eine Demenzerkrankung in den Mittelpunkt seines umstrittenen Textes stellt. Vielmehr entzündet sich die Debatte über die autobiographische Erzählung an der Darstellungsweise einer prominenten Persönlichkeit, die im kollektiven Gedächtnis vor allem als großer Redner bekannt gewesen ist und auch im Schatten der Veröffentlichung seiner NSDAP-Mitgliedschaft Ansehen genießt.

\section{Demenz im 21. Jahrhundert - (k)ein Tabu?}

Niemand hat sich getraut, die Diagnose ohne Hoffnung zu stellen, das Tabu aus sechs Lettern klar zu benennen: DEMENZ, die Krankheit, derer man sich noch immer schämt, wenn [...] zur vaskulären Demenz, verursacht durch eine Vielzahl kleiner unbemerkter Schlaganfälle, auch noch ein Anteil Alzheimer kommt. Alzheimer - das Stigma schlechthin. (Jens 47)

So schildert Tilman Jens die Krankheit in seiner autobiographischen Erzählung ${ }^{1}$ Demenz. Abschied von meinem Vater, die den populären Gelehrten und Tübinger Professor für Rhetorik, Walter Jens, betroffen hat. Das Zitat suggeriert, dass es sich bei der Demenz um ein tabuisiertes Krankheitsbild handelt, 
das der Autor bereits im Titel beim Namen nennt. So verwundert es nicht, dass Demenz im oben genannten Zitat in Versalien abgedruckt ist. Fraglich aber ist, ob die Krankheit im Jahr 2009 tatsächlich noch ein Tabu darstellt, das es zu brechen gilt, existieren doch bereits vor der Jahrtausendwende ähnliche autobiographische Angehörigenpathographien ${ }^{2}$ wie etwa John Bayleys Elegy for Iris (1998), in der aus Sicht des Gatten der krankheitsbedingte Gedächtnisabbau der Ehefrau geschildert wird. Der Text „[gilt] international als prominentestes Beispiel eines Alzheimer-Narratives“ (Dieckmann 58).

Angesichts des Jens-Zitates kommt die Frage auf, inwiefern die Thematisierung von Altersdemenz und Alzheimer ${ }^{3}$ bereits einen Tabubruch darstellt. Zweifelsohne haben insbesondere prominente Persönlichkeiten durch ihre medial-öffentliche Wirkung zur Enttabuisierung von Demenz im Allgemeinen und Alzheimer im Besonderes beigetragen, darunter illustre Persönlichkeiten wie etwa Ronald Reagan, Margaret Thatcher, Rita Hayworth, Siegfried Lenz und Walter Jens (Stoffers 1). Genau genommen war es nicht Walter Jens selbst, der seine Demenzerkrankung an die Öffentlichkeit gebracht hat, sondern dessen Sohn Tilman Jens, der sich den Tabubruch zuschreibt. Dabei spielt der prekäre Umstand, dass ein Angehöriger über den demenziellen Vater schreibt, nur in der ethischen Bewertung des Feuilletons eine untergeordnete Rolle. So haben vergleichbare Texte mit ähnlicher Ausgangssituation wie Arno Geigers Der alte König in seinem Exil (2011) und David Wagners Der vergessliche Riese (2019) im Feuilleton deutlich mehr Anerkennung erhalten. ${ }^{4}$

\footnotetext{
${ }^{2}$ Katharina Fürholzer zufolge bezeichnet man „[a]ls ,Pathographie‘ [...] in der Regel die schriftliche Auseinandersetzung mit einer eigenen oder fremden Krankheitserfahrung und der Bedeutung, die dieser Erfahrung im Kontext des eigenen oder fremden Lebens zukommt." Fürholzer versammelt darunter „sowohl fiktionale als auch faktuale (also nichtfiktionale) Texte, sowohl narrative als auch berichtende Formate und [...] sowohl pathographische Schreibarten als auch pathographische Lesarten" (Fürholzer 4).

${ }^{3}$ Der Begriff Demenz hat sich mittlerweile in der Medizin, aber auch - obgleich undifferenzierter - in der Alltagssprache durchgesetzt. Trotz Fortschritten in der Gerontologie werden die Begriffe Demenz und Alzheimer im alltagssprachlichen Umgang häufig synonym verwendet, in der zeitgenössischen Literatur hingegen spiegelt sich eine Differenzierung wider, die nachvollziehbarerweise in der Medizin noch nuancierter betrieben wird. Die Unterscheidung im literarischen Feld zeigt sich beispielsweise in Obermüllers herausgegebener Anthologie Es schneit in meinem Kopf (2006), die den Untertitel Alzheimer und Demenz trägt.

${ }^{4}$ Besonders lobenswert fällt Felicitas von Lovenbergs Rezension aus: „Arno Geigers Buch ist in jeder Hinsicht das Gegenteil desjenigen von Tilman Jens - und damit eine Bestätigung des Satzes, dass ,Charakter die härtere Währung als guter Wille` ist. Es lässt sich über dieses schwierige, schmerzliche Thema kein weniger larmoyantes und egozentrisches Buch denken als der ,Der alte König in seinem Exil.“ (von Lovenberg). Hubert Winkels zieht Geigers Text zwar als Vergleichsfolie heran, macht
} 
An dieser Stelle lohnt ein genauerer Blick auf den Begriff Tabu, der unter Berücksichtigung der Freud'schen Psychoanalyse näher untersucht wird. Mit seiner Studie Totem und Tabu. Einige Übereinstimmungen im Seelenleben der Wilden und der Neurotiker (1912/13), ,vollzieht sich [...] zu Beginn des 20. Jahrhunderts [ein Paradigmenwechsel]“ (Süwolto 10). Freuds Gedanken tragen im Folgenden dazu dabei, die Bedeutung des Tabu-Begriffs von dem inflationären Gebrauch, wie ihn etwa die Medien im Allgemeinen und Tilman Jens im Besonderen verwenden, zu differenzieren. So ist es ein Verdienst des Psychoanalytikers, dass er das Tabu ,aus seinem ursprünglich religiös konnotierten Kontext löst und es in einem gesamtgesellschaftlichen, entsakralisierten Zusammenhang stellt“; somit erhält der Tabu-Begriff eine „kulturstiftende Funktion“ (9). Vor diesem Horizont erweist sich Freuds Tabu-Verständnis als gewinnbringend. Die Fokussierung des Begriffs dient dazu, den Mechanismus des angeblichen Tabubruchs durch Tilman Jens sowie die Debatten im Feuilleton besser nachvollziehen zu können.

Freud zufolge hafte dem Tabu stets etwas an, das es nicht zu übertreten resp. zu berühren gilt, denn es ist sowohl an Personen als auch an Orte, Gegenstände sowie teilweise an Zustände gebunden, „welche Träger oder Quelle dieser geheimnisvollen Eigenschaft sind“ (Freud 315). Obgleich das Tabu seinen Ursprung in gemeinhin primitiven Naturvölkern hat, um beispielsweise Inzest zu vermeiden und überdies eines der „ältesten ungeschriebenen Gesetzeskodex“ ist (311), fungiert es auch heute noch als mentale Grenze, bestimmte Themen zumindest im öffentlichen Diskurs nicht zu tangieren. Freud arbeitet heraus, dass dem Tabu eine ahistorische Dimension innewohnt, indem er eine Analogie zwischen dem Naturvolk der Polynesier und den an Zwangsneurosen leidenden Menschen herstellt. Aus anthropologischer Sicht befinden sich die Ursprünge des Tabus im Unbewusstsein der Menschheit, allerdings ist seine Erscheinungsform vom historischen und persönlichen Kontext abhängig. Die „auffälligste Übereinstimmung der Zwangsverbote [...] mit dem Tabu besteht nun darin, daß diese Verbote ebenso unmotiviert und in ihrer Herkunft rätselhaft sind“ (319). Damit unterscheidet sich das Tabu von einem durch die Legislative ver-

aber essentielle Unterschiede aus: „Bei Arno Geigers Der alte König in seinem Exil hatten wir es mit der Aufgabe zu tun, einen schweren Fall von pathologisch gestörter Kommunikation aus der Perspektive des Sohns staunend auf seine insularen menschlichen Exzentrizitäten abzuhören. Bei David Wagners Demenz verarbeitender Prosa bleiben wir näher an der sprachlichen Alltagserfahrung und damit an einer beinahe schon üblich werdenden sprachlichen und kognitiven Realitätsverzerrung im Alter, deren Auffälligkeiten man gelegentlich als antiorthodox bejahen möchte." (Winkels 13) 
abschiedeten bzw. geschriebenen Gesetz und ist trotz seiner Unmotiviertheit beständig. Gleichwohl ist hinzuzufügen, dass tabuisierte Gegenstände, Personen oder Themen nicht mehr vom Tabu betroffen sind, wenn sie einmal berührt und die Grenze somit übertreten ist. Ein Tabu zu brechen ist, sofern man es topologisch betrachtet, stets eine Überschreitung einer bestimmten Zone oder der Schritt über die rote Linie. Je nach Art und Ausmaß der Erkrankung sind die Betroffenen stigmatisiert. Dieser Gedanke findet sich auch in Susan Sontags Illness as a Metaphor (1978) [Krankheit als Metapher]. Sontag zufolge trifft diese Form der Tabuisierung insbesondere auf Krebs und Tuberkulose oder, präziser formuliert, auf deren Träger zu: „Der Kontakt mit jemandem, der von einer als mysteriöses Übel betrachteten Krankheit befallen ist, gilt unvermeidlich als Vergehen oder gar als Tabuverletzung. Schon dem bloßen Namen solcher Krankheiten wird magische Macht zugeschrieben“ (Sontag 135). Tilman Jens instrumentalisiert den Begriff Demenz, um ein vermeintliches Tabu zu brechen. ${ }^{5}$ In seiner Apologie Vatermord. Wider einen Generalverdacht führt er diese Überlegung weiter aus, indem er anführt, dass „[d]ie Patienten [...] in die Tabuzone des Nicht-Mehr-Appetitlichen gedrängt [werden], alleingelassen, abgeschnitten vom Alltag, von den wenigen Resten der Erinnerung, die ihnen noch geblieben sind“ (Jens 125). Jens verschränkt damit implizit den Gedankengang Sontags mit der Tabuisierung der Demenzerkrankung und zeichnet überdies ein ausgesprochen finsteres Bild dementer Menschen, die nicht mehr adäquat am gesellschaftlichen Leben teilhaben können. „Daraus ergibt sich das Paradox, dass gerade das erkennbare, das gegenwärtige Leben einer Person mit Demenz die Differenz zum vorherigen Leben offenkundig werden lässt und ihr aktueller Zustand ein Verlustempfinden begünstigt - eine lebende Person wird betrauert, gerade weil sie noch lebt“ (Glasenapp 148).Im Fall von Walter Jens aber kommt noch eine weitere Komponente hinzu, da sein Nachlassen mit der autobiographischen Erzählung nun einer Öffentlichkeit präsentiert wird, worin dem Feuilleton zufolge Jens' eigentliche Grenzüberschreitung besteht.

\footnotetext{
${ }^{5}$ In diesem Zusammenhang ist auch Jens' umstrittene Metaphorisierungsstrategie zu erwähnen. So stellt er die Diagnose Demenz in einen kausalen Zusammenhang mit Walter Jens' NSDAP-Mitgliedschaft, die 2003 publik wurde und ,versucht [...] das Vergessen qua Demenz als ein Vergessen-Wollen zu plausibilisieren“ (Vedder 274). Für Christiane Weller ist diese Strategie psychoanalytisch motiviert: „Für den Erzähler Jens kommt sich der Vater in diesem Verschweigen der Vergangenheit selbst abhanden; die Demenz ist Ausfluss dieser Weigerung, sich den Vorwürfen zu stellen“ (Weller 282). Besonders Iris Radisch findet Anstoß daran: Der Zusammenhang sei „so weit entfernt von jeder medizinischen Vernunft, dass man ihn [...] fast schon literarisch nennen kann" (Radisch 2009). Vgl. zu Jens’ Demenz-Metaphern Dieckmann 103-5.; vgl. ferner Schröder 2017.
} 
Die Tabuisierung betrifft eher den Patienten als die Krankheit. Oder drastischer formuliert: Sie betrifft den Sterbenden. In seinembekannten Essay Über die Einsamkeit der Sterbenden in unseren Tagen von 1982 beschreibt Norbert Elias den zeitgenössischen Umgang mit alternden und sterbenden Menschen. In diesem Zusammenhang nimmt er auch Bezug auf die historische Dimension des Alter(n)s zurück bis in die Antike, worauf er seine Kerngedanken einer Verdrängungsstrategie des Todes in der heutigen Gesellschaft aufbaut. Er verleiht dem Begriff der Verdrängung zwei Bedeutungsebenen: „Wenn man heute von der ,Verdrängung' des Todes spricht, so gebraucht man diesen Begriff, wie mir scheint, in einem doppelten Sinne. Man kann dabei eine, Verdrängung auf der individuellen und auf der sozialen Ebene im Auge haben“ (Elias 18). Auch wenn Elias' Überlegungen nicht im Licht demenzieller Erkrankungen entstanden sind, haben sie an ihrer Aktualität nichts eingebüßt.

Relevant ist darüber hinaus der Aspekt, dass Krankheiten nicht aus medizinischer Sicht als mysteriöses Übel gelten. Vielmehr werden sie durch medizinisch-moralische Diskurse, die sich etwa auch in der Literatur und anderen Medien widerspiegeln, konstruiert. Was Sontag als „mysteriöses Übel“ bezeichnet, ist bei Freud „eine dämonische Macht“ (Freud 317), die dem Tabu bzw. der tabuisierten Krankheit inhärent ist. Tilman Jens instrumentalisiert diese vermeintliche Tabuisierung und stellt diese diskursiv her, indem er den Begriff, wie oben bereits ausgeführt, in Versalien dem Lesepublikum näherbringen will. Bemerkenswerterweise geht die Verdrängung Jens zufolge primär von den Ärzten aus:

Doch die Aura der Prominenz, die vielen persönlichen Gespräche mit dem Patienten, all die in Dankbarkeit gewidmeten Bücher, die seit Jahren die Regale von vielen der behandelnden Ärzte schmücken, scheinen im Fall meines Vaters den professionellen Blick der Mediziner zu vernebeln. Sie bagatellisieren, verbreiten noch immer Optimismus. (Jens 49)

Jens greift das öffentliche Bild seines Vaters auf und betont die Fallhöhe einer prominenten, geistigen Persönlichkeit, der im Stadium der Krankheit noch immer so viel Autorität innewohnt, dass die Ärzte dies nicht sehen wollen. Diese Fallhöhe stellt Jens etwas später im Text drastisch dar. Das Bild des angesehenen Intellektuellen Walter Jens wird der Darstellung des kranken Vaters mit infantilen Verhaltensweisen ein weiteres Mal gegenübergestellt: 
Walter Jens, der unbequeme Denker aus Tübingen, der Redner der Republik, als stammelndes Menschenkind mit dem Babyphon am Bett, da hüllt man sich lieber in Schweigen, als ob dies letzte Kapitel eines langen, reichen und wortreich geführten Lebens ehrenrührig wäre, eine Schande, die es unter den Teppich zu kehren gilt. (49)

Mit der Demenz wird das „Primat der Vernunft und des autonomen Subjekts als rational agierende Einheit“, das zweifellos auf die Persönlichkeit des Rhetorik-Professors Walter Jens zu übertragen ist, „zunehmend kritisch hinterfragt“" (Kuhlmey und Kuhlmey 270). An der Fallhöhe der prominenten Persönlichkeit entzündet sich demnach nicht in erster Linie ein Diskurs über die Demenzerkrankung, sondern über den Umgang mit hochaltrigen Menschen, die alters- und krankheitsbedingt geistig abbauen. Die Rezensionen und Verrisse im Feuilleton, die Tilman Jens' Autobiographie scharf verurteilen, indem sie ihm beispielsweise einen „Denkmalsturz“ (Radisch) ${ }^{6}$ oder gar einen Vatermord unterstellen, bestätigen die These, dass nicht die Literarisierung oder Medialisierung der Demenz und Alzheimer-Krankheit ein Tabubruch darstellt, sondern die Art und Weise, wie Tilman Jens seinen Vater zeichnet: „Nachts, wenn der große Hunger kommt und das Schlafmittel keine Ruhe mehr gibt, strampelt er sich frei.“ (Jens 11) In seiner Rezension unterstellt Gert Ueding, ehemaliger Doktorand von Walter Jens, dem Autor, dass es sich um eine Abrechnung mit dem kranken Vater handele und dass das „Werk intime, besser verborgene Geheimnisse aus dem Leben von Walter Jens preis[gebe]“ (Ueding). Die Kontrastierung von öffentlichem Leben und intimsten Szenen des Professors sind für das Feuilleton derartig provokant, dass die Schilderungen offensichtlich nur schwer auszuhalten sind, da Walter Jens als „Vater der Rhetorik“ sowie als akademische Größe im kulturellen Gedächtnis gleich einem Monument fest verankert ist. Karl-Josef Kuschel lobpreist Walter Jens mit den Worten:

Er verkörpert in Deutschland das, was man im Amerikanischen einen ,public intellectual' nennt, jemanden also im Raum der Kultur, der nicht nur den Geheimcode seiner Zunft spricht, sondern aufgrund seiner Sach- und Sprachkompetenz in der allgemeinen Öffentlichkeit wahrgenommen und verstanden wird und aufgrund seines Selbstverständnisses Wissenschaftsfragen als Lebensfragen transparent zu machen versteht. (Kuschel 11)

\footnotetext{
${ }^{6}$ Radisch kommt am Ende ihrer Rezension zu folgendem Urteil: „Und so ist der Denkmalsturz, als dieses empörende Buch in Erinnerung bleiben wird, am Ende ein Beweis einer enttäuschten, irregeleiteten Liebe." (Radisch 2009).
} 
Über die Alzheimer- und Demenzerkrankung macht der Theologie-Professor allerdings keine Angaben, was Tilman Jens u. a. kritisch bemerkt: „Selbst Karl-Josef Kuschels sonst so genaues Porträt [...] macht einen weiten Bogen um die Krankheit, die meinem Vater die Sprache nahm“ (Jens 49).

In Uedings Rezension (2009) wird der Begriff Demenz lediglich einmal durch den Buchtitel erwähnt. Dies ist insofern bemerkenswert, als tatsächlich nicht die Thematisierung der Demenzerkrankung für den Verriss ausschlaggebend ist. Auffällig oft hingegen verwendet der Rezensent den Begriff Vater, der dreiundzwanzigmal in der Rezension vorkommt. Bereits die Quantität der verwendeten Begriffe verdeutlicht den inhaltlichen Schwerpunkt der Kritik. Die Zahlen weisen nicht auf die literarische Behandlung altersbedingter Krankheiten hin, sondern auf das von Tilman Jens gezeichnete Vaterbild, das Ueding heftig verurteilt: „Infam ist die Methode und infam das Porträt, das dabei heraus kommt" (Ueding). Wenn Radisch in ihrer Kritik metaphorisch von einem „Denkmalsturz“ spricht (Radisch), unterstellt sie dem Autor eine Entwertung der Symbolfigur und rhetorischen Legende Walter Jens (Geyer 2009). Der Begriff des Denkmals impliziert eine zuvor stattgefundene Überhöhung des Vaters, der im kollektiven Gedächtnis auf einem Sockel stand. Aber auch andere Stimmen des Feuilletons greifen zu rhetorischen Mitteln und nutzen Sprachbilder, um die Fallhöhe Walter Jens' in Demenz. Abschied von meinem Vater eklatant herauszustellen, wobei sie den Zweck verfolgen, auf die für sie unangemessenen Darstellungsstrategien der autobiographischen Erzählung zu verweisen und Tilman Jens als Tabubrecher darzustellen. So sieht vor allem der Focus-Kritiker Jobst-Ulrich Brand in den Verrissen eine schleichende Tabuisierung des Sohnes Tilman Jens, der seinen Vater denunziere (Radisch). In Vatermord. Wider einen Generalverdacht wehrt sich Jens gegen die Vorwürfe des Feuilletons und folgt Brands These, dass er selbst eine Person sei, die tabuisiert werde. Der Gedanke, dass eine Person tabuisiert sei, mag auf den ersten Blick befremdlich anmuten, ist aber ein relevanter Aspekt bei Freud und lässt sich psychoanalytisch plausibilisieren: „Wir wissen, ohne es zu verstehen, wer das Verbotene tut, das Tabu übertritt, wird selbst tabu“ (Freud 324). Das Tabu nimmt somit eine weitere Funktionsstelle ein, die zunächst nichts mehr mit der eigentlichen Krankheit zu tun hat, sondern vielmehr mit der Tatsache, dass Tilman Jens beschreibt, wie die Demenz „aus Geistesgrößen hilflose Kreaturen macht [...]“ (Brand 2009). Der Autor selbst wird Brand zufolge zum Tabuisierten im Sinne Freuds: „Der Mensch, der ein Tabu übertreten hat, wird selbst tabu, weil er die gefährliche 
Eignung hat, andere zu versuchen, daß sie seinem Beispiel folgen“" (Freud 324). Demnach handelt es sich um einen ausgesprochen relevanten Aspekt, der im Rahmen der anthropologischen Lesart einmal mehr Berücksichtigung finden soll. Im Feuilleton wird Tilman Jens des literarischen Patrizids bezichtigt und begeht damit den Tabubruch schlechthin. Das Prekäre dabei ist, dass Walter Jens zum Zeitpunkt der Textveröffentlichung noch gelebt und nicht geahnt hat, welche teils intimen und drastischen Schilderungen sein Sohn verarbeitet hat. Vor diesem Hintergrund kommt erschwerend hinzu, dass „der bekannte Rhetoriker stets für einen menschenwürdigen Umgang mit Kranken und vor allem deren selbstbestimmtes Ende plädiert [hatte]“, worin zusätzliches „Provokationspotenzial der Demenz-Darstellung“ liegt (Dieckmann 97).

\section{Die Fallhöhe eines Professors}

Dass Jens' Verteidigungsschrift ausgerechnet den ebenso semantisch wie historisch starken Begriff Vatermord trägt, ist kein Zufall, sondern ein durchdachter Zug des belesenen Journalisten, wodurch er eine psychoanalytische Lesart seiner Vaterbindung geradezu herausfordert. Jens liefert eine simpel erscheinende Antwort auf die durchaus emotional konnotierten Verrisse im Feuilleton: „Die Republik erträgt nun einmal keine Ikonen mit Makel“ (Jens 139) lautet seine angriffslustige These. Er reagiert damit direkt auf die heftig ausfallenden Kritiken und führt philosophische Gedankengänge etwa Nietzsches und Thomas Manns implizit weiter.

In der deutschen Kulturgeschichte des 20. Jahrhunderts ist ein durchaus vergleichbares Phänomen zu verzeichnen, das an dieser Stelle zum besseren Verständnis der Debatte um die autobiographische Erzählung umrissen wird. In seinem Vortrag Nietzsches Philosophie im Lichte unserer Erfahrungen von 1948 arbeitet Thomas Mann das Verhältnis zwischen Krankheit und Persönlichkeit heraus, welches durchaus allegorisch auf Jens' Demenz. Abschied von meinem Vater und die Debatte im Feuilleton zutrifft. Thomas Mann reflektiert den Tod Nietzsches und setzt dessen Schicksal mit dem Geniebegriff in Zusammenhang: „Was war es, was Nietzsche ins Unwegsame trieb, ihn unter Qualen dort hinaufgeißelte und ihn in den Martertod am Kreuz des Gedankens sterben ließ? Sein Schicksal - und sein Schicksal war sein Genie. Aber dieses Genie hat noch einen anderen Namen. Er lautet: Krankheit [...]“ (Mann 8-9). Für Mann hat der Geniebegriff im Licht von Nietzsches Schicksal und Tod zwei Seiten: Einerseits 
den Tod, andererseits die Krankheit, deren semantischen Gehalt er schließlich spezifiziert:

[...] Krankheit - dies Wort nicht in dem vagen Sinn und allgemeinen Sinn genommen, in welchen es sich so leicht mit dem Begriff des Genies verbindet, sondern in einem so spezifischen und klinischen Verstande, daß man sich wiederum dem Verdacht des Banausentums und dem Vorwurf aussetzt, man wollte die schöpferische Lebensleistung eines Geistes damit entwerten, der als Sprachkünstler, Denker, Psychologe und die ganze Atmosphäre einer Epoche verändert hat. (9)

Thomas Mann antizipiert offenbar Vorwürfe, wenn er den einflussreichen Philosophen Nietzsche ungeschönt mit einer klinischen Krankheit assoziiert. Nietzsche hatte ein besonderes Verhältnis zur Krankheit, das unter anderem dem Zeitgeist im späten 19. Jahrhundert geschuldet war, in dem insbesondere in gehobenen intellektuellen Kreisen Krankheit als Vervollkommnung des Künstlergeistes idealisiert worden ist. Dies schlägt sich etwa in Manns Roman Der Zauberberg (1924) nieder, in denen die Figuren zwar reihenweise und elendig an Tuberkulose sterben, aber gleichzeitig mit der Aura des Mysteriösen versehen werden. Dies ist nicht überraschend, denn

[o]bwohl es in der zweiten Hälfte des letzten Jahrhunderts eine gewisse Reaktion gegen den romantischen Kult der Krankheit gab, bewahrte die $\mathrm{Tb}$ die meisten ihrer romantischen Attribute - Zeichen einer überlegenen Natur, kleidsame Anfälligkeit - bis zum Ende des Jahrhunderts und ein gutes Stück in unseres hinein. (Sontag 37)

$\mathrm{Zu}$ berücksichtigen ist allerdings, dass im Zauberberg ausschließlich infektiöse Krankheiten im Mittelpunkt stehen, worin sie sich von demenziellen Erkrankungen unterscheiden. Die Krankheit selbst bleibt für den Schriftsteller zunächst etwas „bloß Formales“ (Mann 9). Schließlich formuliert der Schriftsteller einen höchst entscheidenden Satz: „Es kommt darauf an, wer krank ist: ein Durchschnittsdummkopf, bei welchem die Krankheit des geistigen und kulturellen Aspektes freilich entbehrt, oder ein Nietzsche, ein Dostojewski [Hervorheb. im Orig.]“ (ebd.). Dies fügt sich an die eingangs aufgestellte Bedingung von Pathologiegeschichten, dass jede Krankheit eine Projektionsfläche im Sinne einer realen Persönlichkeit oder literarische Figur benötigt, um dargestellt zu werden. Doch Persönlichkeiten oder Figuren werden nicht nur auf ihre Funktion reduziert, eine Krankheitsgeschichte zu transportieren; vielmehr manifes- 
tiert sich in ihnen eine Persönlichkeitsentwicklung. Mit dem ethisch durchaus fragwürdigen Satz, es komme darauf an, wer krank ist, formuliert Thomas Mann genau das, was in der Feuilletondebatte um Tilman Jens' Demenz. Abschied von meinem Vater entbrannt ist: Die Frage, ob man die Persönlichkeit Walter Jens im Zustand seines geistigen und körperlichen Abbaus so intim schildern darf.7 An den autobiographischen Erzählungen, wie sie von Jens, Geiger und Wagner vorliegen, entzünden sich ethische Fragen, die insbesondere im Kontext von Demenz. Abschied von meinem Vater im Feuilleton diskutiert werden:

Durch die Intimität der in ihr dargestellten Beziehungen und die damit verbundene Grenzaufhebung von Fremdbiographie und Autobiographie stellen sich bei Angehörigenpathographien stets ethische Fragen: Inwiefern hatte die kranke Person Einfluss auf ihre eigene Repräsentation? Wie verhält sich das entstandene Porträt zu den Vorstellungen und dem Wohl der porträtierten Person? Welcher Schaden kann durch eine Fehlrepräsentation entstehen, etwa mit Blick auf die Privatheit, die Reputation und auch die personale Integrität des Porträtierten? (Fürholzer 8)

Im Gegensatz zu Jens' Demenz. Abschied von meinem Vater sind Geigers und Wagners autobiographische Erzählungen deutlich lobender besprochen worden. Ein weiterer wichtiger Aspekt, den Mann heraushebt, ist die medizinisch-pathologische Wahrheit, die naturalistische Seite, welche hinter Darstellungsverfahren von Krankheiten zum Ausdruck kommt: „[...] und wer die Wahrheit als Ganzes liebt und willens ist, ihr unbedingt die Ehre zu geben, wird nicht aus geistiger Prüderie irgendeinen Gesichtspunkt verleugnen, unter dem sie gesehen werden kann“ (Mann 9) Mit einem konkreten Beispiel veranschaulicht Mann seinen Gedanken:

Man hat es dem Arzte Möbius sehr verübelt, daß er ein Buch geschrieben hat, worin er die Entwicklungsgeschichte Nietzsches als die Geschichte einer progressiven Paralyse fachmännisch darstellt. Ich habe an der Entrüstung darüber nie teilnehmen können. Der Mann sagt, auf seine Weise, die unbestreitbare Wahrheit. (9)

\footnotetext{
${ }^{7}$ Würdigend hingegen wird im Feuilleton die Briefsammlung Langsames Entschwinden. Vom Leben mit einem Demenzkranken (2016) von Inge Jens wahrgenommen, da die Briefe um den dementen Ehemann Walter Jens drei Jahre nach dessen Tod veröffentlicht worden sind und im Vordergrund Reflexionen über den Umgang mit pflegebedürftigen Menschen stehen (Jaiser 2016).
} 
Bemerkenswert ist, dass ein solches affirmatives Urteil über Krankheitsdarstellungen durchaus vergleichbar ist mit jenen wenigen Feuilleton-Kritiken, die Jens' Demenz. Abschied von meinem Vater ein Lob aussprechen. ${ }^{8}$ Thomas Mann wäre möglicherweise ein Verfechter des Textes gewesen und spricht mit der eingeschobenen, aber nicht minder relevanten Formulierung „auf seine Weise" den Darstellungsverfahren einen enormen Spielraum zu. Einen Tabubruch würden die intimen Beschreibungen des kranken Walter Jens für Thomas Mann keineswegs darstellen.

Der Gedanke, es komme darauf an, wer krank sei, impliziert eine Hierarchie, die nicht nur das asymmetrische Verhältnis von Gesunden und Kranken diskursiv bestärkt, sondern der obendrein die Dimension eines Ordnungs- und Machtmusters unter kranken Männern zukommt. Prekär ist im Fall von Walter und Tilman Jens, dass die Hierarchie zwischen Vater und Sohn umgekehrt wird. ${ }^{9}$ Überdies „etabliert Thomas Mann nicht nur eine Art Patientenhierarchie, sondern in gleichem Maße eine Geschlechterhierarchie, denn die Genialisierung bleibt ausschließlich Männern vorbehalten. Die Krankheit fungiert in diesem Verständnis nicht als Bedrohung der Männlichkeit, sondern dient deren Veredelung" (Schuhen 14). Thomas Mann spricht ausschließlich über Männer und unterscheidet deutlich zwischen geistigen Größen und solchen, die keinerlei Beachtung in intellektuellen Kreisen finden. Durch den despektierlichen Ausdruck „Durchschnittsdummkopf“, den er bekannten intellektuellen Persönlichkeiten wie Nietzsche und Dostojewski bewusst als Kontrastfolie entgegensetzt, impliziert Mann, vielleicht ohne sich dessen vollkommen bewusst zu sein, dass Männer, die einer niedrigeren sozialen Klasse angehören, im Falle einer Erkrankung weniger zu beachten sind als solche, deren Fallhöhe immens ist. Plakativ formuliert: Stellt man einen Arbeiter als geistig oder körperlich krank dar, so erregt dies keinen Anstoß; stellt man eine geistige Größe wie Nietzsche als geistig oder körperlich krank dar, so ruft dies Entrüstung hervor. Dieser Gedanke manifestiert sich heute bemerkenswerterweise in der medialen Verarbeitung von an Demenz erkrankten Persönlichkeiten oder Figuren, wie deutlich am Beispiel

\footnotetext{
${ }^{8}$ So hebt etwa Brand in seiner Kritik resümierend Tilman Jens' Mut hervor: „Tilman Jens' Buch ist erschreckend - so erschreckend, wie gute Reportagen bisweilen sind. Dass er beschreibt, welche verheerenden Folgen Alzheimer für die Betroffenen hat, wie die Krankheit ganze Familien zerrütten kann, wie sie aus Geistesgrößen hilflose Kreaturen machen, ist mutig“ (Brand 2009).

${ }^{9}$ Obgleich Inge Jens in ihren veröffentlichten Briefen über ihren dementen Ehemann schreibt, ist sie allein wegen des Alters ihrem Mann ebenbürtig, „wohingegen die Perspektive eines Kindes auf einen Elternteil immer durch ein Gefälle geprägt ist“"(Dieckmann 196).
} 
von Walter Jens zu sehen ist. Aber auch in der Fiktion sind es überwiegend Charaktere, die aufgrund ihrer sozialen Schicht - meist gehobene Mittel- oder Oberschicht - eine enorme Fallhöhe haben, wie beispielsweise der von Klaus Maria Brandauer verkörperte, langsam dement werdende Kunsthistoriker im Fernsehfilm Die Auslöschung, inszeniert von Nikolaus Leytner. Während der Protagonist zu Beginn der Handlung seine Eloquenz während eines Vortrages eindrucksvoll unter Beweis stellt, irrt er am Ende in einem verwahrlosten $\mathrm{Zu}$ stand in seinem Büro umher. Der Fernsehfilm hat viel Lob für die Darstellung der Demenzerkrankung bekommen, weil er anders als Jens' Demenz im geschützten Raum der Fiktion spielt. ${ }^{10}$

\section{Schlussbemerkung}

Der Beitrag hat die These verfolgt, dass nicht die Thematisierung von Demenz - wie Tilman Jens in Demenz. Abschied von meinem Vater behauptet ein Tabu ist. „Warum schützt den Vater niemand vor seinem Sohn?" (Radisch). Diese Frage steht paradigmatisch für die Kontroverse um Tilman Jens’ Text und zeigt, dass Walter Jens auch nach seiner Demenz-Diagnose erstaunlich präsent gewesen ist, allerdings nicht als Patient, sondern als intellektuelle Größe. Die Debatte verdeutlicht, dass Jens' ambivalente Darstellungsstrategie zwischen Verklärung des Vaters einerseits und der drastischen Schilderungen des dementen Walter Jens andererseits im Feuilleton als unethisch und anstößig empfunden wird. Nicht die Thematisierung der Demenz ist das Tabu, wie Tilman Jens der Leserschaft nahebringen will. Vielmehr liegt das Tabu in der Art und Weise, wie er den Verfall seines Vaters und die den krankheitsbedingten Gedächtnisabbau begleitende Regression schildert. Die zahlreichen empörten Stimmen der Literaturkritik sind symptomatisch für diese Beobachtung.

„Der Patient ist kein Mann des Intellekts mehr“ (Schröder 297). Auch dieser treffend formulierte Satz ist eine Diagnose, die so schwer auszuhalten ist, dass im Feuilleton Angriffe gegen die Person Tilman Jens laut werden. Die mediale Aufmerksamkeit nutzt der Autor, indem er mit der Verteidigungsschrift Vatermord. Wider einen Generalverdacht reagiert und die Kontroverse aufgreift. Nunmehr stilisiert er sich als Opfer, als Tabu-Brecher, der wiederum tabuisiert

${ }^{10}$ Eine ähnliche Handlungsstruktur weist der oscarprämierte Kinofilm Still Alice (2014), inszeniertvon Richard Glatzer und Wash Westmoreland, auf. Eine Ausnahme bildet der deutsche Fernsehfilm Mein Vater (2003) von Andreas Kleinert, in dem der Protagonist, dargestellt von Götz George, einen Busfahrer mimt, der an Alzheimer erkrankt. 
gehöre. Die Verrisse im Feuilleton sind insofern bemerkenswert, als vergleichbare autobiographische Erzählungen wie Geigers Der alte König in seinem Exil und Wagners Der vergessliche Riese überwiegend lobenswert besprochen worden sind, ja, Geigers Text gilt im deutschsprachigen Raum als „Schlüsseltext“ der zeitgenössischen Demenz-Literatur (Dieckmann 27). Anders als bei Walter Jens, der „zu den wichtigen public intellectuals der Bonner Bundesrepublik [gehörte]“ (Knape 18), handelt es sich bei den Vätern von Geiger und Wagner allerdings nicht - und das ist der bedeutende Unterschied - um prominente Persönlichkeiten. Vor diesem Hintergrund wurden im Beitrag Thomas Manns Überlegungen aus Nietzsches Philosophie im Lichte unserer Erfahrungen herangezogen, um die überwunden geglaubte These, es komme darauf an, wer krank sei, im Licht der demenziellen Erkrankung von Walter Jens zu aktualisieren und bestätigen.

Es ist erstaunlich, dass Tilman Jens' Text lange nicht von der literaturwissenschaftlichen Forschung beachtet worden ist, sieht man von Wellers psychoanalytischer Lesart und Dieckmanns Analyse ab. Denn trotz der umstrittenen Darstellungsstrategien und der Debatte im Feuilleton handelt es sich immerhin um einen der ersten deutschsprachigen Texte, die aus Sicht eines Sohnes über den noch lebenden dementen Vater schreiben. Auch wenn Geiger und Wagner sich Demenz. Abschied von meinem Vater womöglich nicht als gelungenes Vorbild genommen haben, so ist festzuhalten, dass Jens als einer der ersten Autoren mit seinem Text ungeachtet aller ethischen Vorbehalte einen wichtigen Beitrag in der deutschsprachigen Demenz-Literatur aus Angehörigen-Perspektive geleistet hat. Die augenfälligen Ambivalenzen werden von Geiger und Wagner nicht wiederholt; dies gilt auch für künftige Verarbeitungen aus der Sicht von Söhnen (oder Töchtern). Von dementen Vätern geht offensichtlich eine gewisse Faszinationskraft aus, die es schreibend zu verarbeiten gilt. Dann gilt es wieder danach zu fragen, ob es sich um eine berühmte Persönlichkeit oder einen unbekannten Vater handelt.

\section{Literaturverzeichnis}

Bayley, John. Elegy for Iris. Picador, 1998.

Brand, Jobst-Ulrich: „Tragödie einer Geistesgröße“. Focus, 20. 02. 2009. https://www.focus.de/kultur/buecher/brands-buecher/walter-jens-tragoedie-einer-geistesgroesse_aid_373265.html/ Abruf: 16. Mai 2021.

Dieckmann, Letizia. Vergessen erzählen. Demenzdarstellungen der deutschsprachigen Gegenwartsliteratur. Transcript, 2021. 
Freud, Sigmund. „Totem und Tabu. Einige Übereinstimmungen im Seelenleben der Wilden und der Neurotiker." Fragen der Gesellschaft-Ursprünge der Religion. Alexander Mitscherlich et al. (Hg.). S. Fischer, 1974, S. 287-444.

Fürholzer, Katharina. Das Ethos des Pathographen. Literatur- und medizinethische Dimensionen von Krankenbiographien. Universitätsverlag Winter, 2019.

Geiger, Arno. Der alte König in seinem Exil. Hanser, 2011.

Geyer, Christian und Walter Jens: Es muss ja nicht gerade heute passieren." Frankfurter Allgemeine Zeitung, 22.02.2009. http://www.faz.net/aktuell/feuilleton/buecher/walterjens-es-muss-ja-nicht-gerade-heute-passieren-1770918.html.Abruf: 16. Mai 2021.

Glasenapp, Nicolai. „Lebt man in Vergessenheit. Zum Konnex von Sterben, Tod und Demenz in der deutschsprachigen Gegenwartsliteratur." Limbus. Australisches Jahrbuch für germanistische Literatur- und Kulturwissenschaft8, 2015, S. 147-65.

Jaiser, Ingeborg. „Anwesende Abwesenheit.“ Titel Kulturmagazin, 23.04.2016. https://itel-kulturmagazin.net/2016/04/22/inge-jens-langsames-entschwinden/?cn-reloaded=1. Abruf: 26.05.2021.

Lovenberg, Felicitas von: „Wenn einer nichts weiß und doch alles versteht.“Frankfurter Allgemeine Zeitung, 04.02.2011, http://www.faz.net/aktuell/feuilleton/buecher/rezensionen/belletristik/arno-geiger-der-alte-koenig-in-seinem-exil-wenn-einer-nichtsweiss-und-doch-alles-versteht-1597380.html. Abruf: 16.05.2021.

Jens, Inge. Langsames Entschwinden. Vom Leben mit einem Demenzkranken. Rowohlt, 2016. Jens, Tilman. Demenz. Abschied von meinem Vater. Gütersloher Verlagshaus, 2009.

Jens, Tilman. Vatermord. Wider einen Generalverdacht. Gütersloher Verlagshaus, 2010.

Knape, Joachim. „Walter Jens als öffentliche Orientierungsfigur. Rede aus Anlass des 90. Geburtstags.“ Walter Jens. Redner - Schriftsteller - Übersetzer. Joachim Knape et. al.(Hg.), Narr Francke Attempto, 2014, S. 9-21.

Kuhlmey, J. und Adelheid Kuhlmey. „Literatur und Medizin: die Demenz." Zeitschrift für Gerontologie und Geriatrie Jg. 46, Nr. 3, 2013, S. 270-76.

Kuschel, Karl-Josef. Walter Jens. Literat und Protestant. Narr Francke Attempto, 2008.

Mann, Thomas. Der Zauberberg. S. Fischer, 2002.

Mann, Thomas. Nietzsches Philosophie im Lichte unserer Erfahrungen. Suhrkamp, 1948.

Obermüller, Klara (Hg.). Es schneit in meinem Kopf. Erzählungen über Alzheimer und Demenz. Nagel \& Kimche, 2006.

Radisch, Iris. Der Mann seines Lebens. Zeit Online 09 (2009). URL: $\quad$ http://www.zeit. de/2009/09/L-Jens. Abruf: 17. Mai 2021.

Schröder, Berenike. „Den Tod überschreiben. Demenz in der deutschsprachigen Literatur der Gegenwart." Krankheit, Sterben und Tod im Leben und Schreiben europäischer Schriftsteller. Bd 2. Das 20. und 21. Jahrhundert. Roland Berbig et. al. (Hg.), Königshausen \& Neumann, 2017, S. 287-301.

Schuhen, Gregor. „Bedrohte oder veredelte Männlichkeit? Phantasmagorien des Pathologischen bei Thomas Mann und André Gide.“ Ambivalente Männlichkeit(en). Mas- 
kulinitätsdiskurse aus interdisziplinärer Perspektive. Uta Fenske und Gregor Schuhen (Hg.). Verlag Barbara Budrich, 2012, S. 213-32.

Sontag, Susan. Krankheit als Metapher. Hanser, 1978.

Stoffers, Tabea. Demenz erleben. Innen- und Außenansichten einer vielschichtigen Erkrankung. Springer, 2016.

Süwolto, Leonie. „Ästhetik des Tabuisierten in der Literatur- und Kulturgeschichte. Eine Einleitung. “ Ästhetik des Tabuisierten in der Literatur- und Kulturgeschichte. Leonie Süwolto (Hg.), Universitätsbibliothek Paderborn, 2017, S. 8-22.

Ueding, Gert. „Tilman Jens begräbt den lebenden Vater.“ Die Welt, 18.02.2009, https://www. welt.de/kultur/article3220547/Tilman-Jens-begraebt-den-lebenden-Vater.html. Abruf: 30.09.2021.

Vedder, Ulrike. „Erzählen vom Zerfall. Demenz und Alzheimer in der Gegenwartsliteratur.“ Zeitschrift für Germanistik Jg. 22, H. 2, 2012, S. 274-90.

Wagner, David. Der vergessliche Riese. Rowohlt, 2019.

Weller, Christiane. „Vom Erinnern und Vergessen alter Väter. Ödipale Vatergespräche bei Thomas Harlan, Tilman Jens und Arno Geiger." Deutsche Gegenwarten in Literatur und Film. Tendenzen nach 1989 in exemplarischen Analysen. Olivia C. Díaz Pérez et. al.(Hg.), Stauffenberg, 2017, S. 277-88.

Winkels, Hubert. „Der vergessene Sohn.“ Zeit Literatur, Nr. 42, 2019, S. 10-11.

\section{Filmografie}

Die Auslöschung. Reg. Nikolaus Leytner. Darst. Klaus Maria Brandauer und Martina Gedeck. Mona Film, 2013.

Mein Vater. Reg. Andreas Kleinert. Darst. Götz George, Klaus J. Behrendt, Ulrike Krumbiegel und Christine Schorn. Colonia Media, 2003.

Still Alice. Reg. Richard Glatzer/Wash Westmoreland. Darst. Julianne Moore, Alec Baldwin und Kristen Stewart. BSM Studio, 2014. 


\title{
TABOO BREAKING AS A STRATEGY? ON THE REPRESENTATION OF DEMENTIA IN TILMAN JENS' DEMENZ. ABSCHIED VON MEINEM VATER (2009)
}

\begin{abstract}
Michael FASSEL

Philosophische Fakultät, Universität Siegen, Germany

Adolf-Reichwein-Str. 2, 57068 Siegen

michael.fassel@uni-siegen.de
\end{abstract}

In the context of this autobiographical narrative, in which Tilman Jens describes the mental decline of his father Walter, the author designates himself as a taboo-breaker since according to him dementia contains an inherent stigma. In fact, it is possible to consider Jens a pioneer of German-language literature on dementia, and the outrageous reactions by arts sections of several national newspapers may confirm this central thesis at first glance. This paper takes a closer look at Jens' text as well as the critical reviews, and aims to show that it is not the thematisation of dementia, but rather the way in which the prominent personality of Walter Jens is portrayed that triggers ethical concerns. Against this background, this analysis draws on the understanding of taboo according to Sigmund Freud, firstly to determine whether and to what extent Jens is breaking a taboo. Building on this, the article pursues the thesis that it is above all the depiction of the illness-related memory loss of a prominent intellectual like Walter Jens that is the real scandal, while comparable dementia narratives are discussed much more positively. In the light of the demented Walter Jens, Thomas Mann's consideration that it depends on who is ill proves to be decidedly relevant.

Keywords: dementia, taboo, illness, pathography, father, Tilman Jens, Demenz Abschied Von Meinem Vater 


\title{
RAZBIJANJE TABUA KAO STRATEGIJA? O PRIKAZU DEMENCIJE U DJELU DEMENZ. ABSCHIED VON MEINEM VATER (2009.) TILMANA JENSA
}

\author{
Sažetak

Michael FASSEL
Philosophische Fakultät, Universität Siegen, Njemačka
Adolf-Reichwein-Str. 2, 57068 Siegen
michael.fassel@uni-siegen.de

U kontekstu autobiografske pripovijesti u kojoj opisuje mentalni krah svojeg oca Waltera, autor Tilman Jens sebi pripisuje ulogu razbijača tabua s obzirom na stav da je demencija inherentno stigmatizirana. Jensa je ustvari moguće smatrati pionirom književnosti o demenciji na njemačkom jeziku, a tu središnju tezu na prvi pogled potvrđuju žestoke reakcije umjetničkih sekcija nekoliko narodnih tiskovina. Ovaj rad pomno razmatra Jensov tekst i njegovu kritiku s ciljem pokazivanja da etički upitna nije tema demencije sama po sebi, već način na koji je prikazana istaknuta ličnost Waltera Jensa. Pritom se analiza oslanja na poimanje tabua prema Sigmundu Freudu, ponajprije radi utvrđivanja otklanja li Jens tabu i u kojoj mjeri. Nastavno na to, zastupa se teza da je ustvari skandalozan prikaz gubitka pamćenja izazvanog bolešću istaknutog intelektualca poput Waltera Jensa, dok se o sličnim pripovijestima o demenciji raspravlja mnogo pozitivnije. S obzirom na dementnog Waltera, misao Thomasa Manna da sve ovisi o tome tko je bolesnik pokazuje se izrazito relevantnom.

Ključne riječi: demencija, tabu, bolest, patografija, otac, Tilman Jens, Demenz. Abschied von meinem Vater 